\title{
Demo Abstract: Identification of LPWAN Technologies using Convolutional Neural Networks
}

\author{
Adnan Shahid*, Jaron Fontaine*, Jetmir Haxhibeqiri*, Martijn Saelens*, Zaheer Khan ${ }^{\dagger}$, Ingrid Moerman*, \\ Eli De Poorter* \\ * IDLab, Ghent University - imec, Ghent, Belgium \\ $\dagger$ CWC, University of Oulu, Finland \\ Email: adnan.shahid@ugent.be
}

\begin{abstract}
This paper demonstrates a Convolutional Neural Network (CNN) based mechanism for identification of low power wide area network (LPWAN) technologies such as LoRA, Sigfox, and IEEE 802.15.4g. Since the technologies operate in unlicensed bands and can interfere with each other, it becomes essential to identify technologies (or interference in general) so that the impact of interference can be minimized by better managing the spectrum. Contrary to the traditional rule-based identification mechanisms, we use Convolutional Neural Networks (CNNs) for identification, which do not require any domain expertise. We demonstrate two types of CNN based classifiers: (i) CNN based on raw IQ samples, and (ii) CNN based on Fast Fourier Transform (FFT), which give classification accuracies close to $95 \%$ and $\mathbf{9 8 \%}$, respectively. In addition, an online video is created for demonstrating the process [1].
\end{abstract}

\section{INTRODUCTION}

The number of Internet of things (IoT) devices are increasing at an unprecedented rate and it is expected that by 2030 there will be around 500 billion devices connected to the internet [2]. To connect all these devices, recently a number of long range IoT technologies have been introduced, collectively referred to as low power wide area networks (LPWANs). The main LPWAN technologies which are very popular in the IoT market are: LoRA [3] and Sigfox [4]. In addition, IEEE has also proposed an amendment for 802.15.4, namely $802.15 .4 \mathrm{~g}$, to operate in sub-1 GHz band [5]. These three technologies operate in unlicensed sub-GHz bands, typically $868 \mathrm{MHz}$ in Europe and $915 \mathrm{MHz}$ in North America. In this work, we consider the operation of LPWAN technologies in Europe, where most technologies need to confirm to a maximum duty cycle regulation of $1 \%$ [6]. However, the proposition can be applied to any region other than Europe.

Since the technologies operate in similar frequency bands, their transmission can interfere with each other. Therefore, it is imperative to identify technologies (or interference in general) and spectrum occupancy information so that the probability of successful communication can be improved. In order to realize this, a spectrum manager is required which can provide upto-date spectrum occupancy and interference information to the participating IoT networks. Within the context of LPWAN technologies operation in Europe, mostly it is assumed in the literature that due to the the duty cycle regulation, there will be negligible interference. Additionally, this is due to the fact that the number of messages that an IoT device can send is typically low. However, as the network density increases due to the increasing popularity of IoT, the interference can no longer be ignored and can result in packet losses by degraded signal-to-interference-plus-noise ratio (SINR).

Traditionally, identification of transmissions is done by rulebased mechanisms such as energy-detection but it is not suitable when multiple co-existence technologies are considered. For such a case, an advanced classification algorithm is required such as cyclostationary feature detection [7]; however, it incurs huge computational complexity. Recent advancement in the field of machine learning has shown tremendous performance in classification problems as it is now possible to identify various complex patterns, which is the case when technologies coexist. Particularly, Convolutional Neural Networks (CNNs) have shown outstanding results in various benchmarks such as image classification [8], modulation classification [9], etc. However, most of these works focus on classification of interference sources in $2.4 \mathrm{GHz}$ rather than LPWAN technologies, which have different behaviors (more narrow bandwidths, longer packet durations, technologies with spreading factors rather than traditional modulations, etc.)

To fill this gap, we aim to demonstrate two CNN based classifiers: (i) CNN based on raw IQ samples, and (ii) CNN based on FFT of the IQ samples, where each does not require domain expertise. Contrary to the traditional identification mechanisms, the former classifier is designed with raw IQ samples while the later with FFT of the IQ samples of the technologies (LoRA, Sigfox, and IEEE 802.15.4g). The reason behind the two classifiers is to have a performance analysis trade-off in different noise conditions.

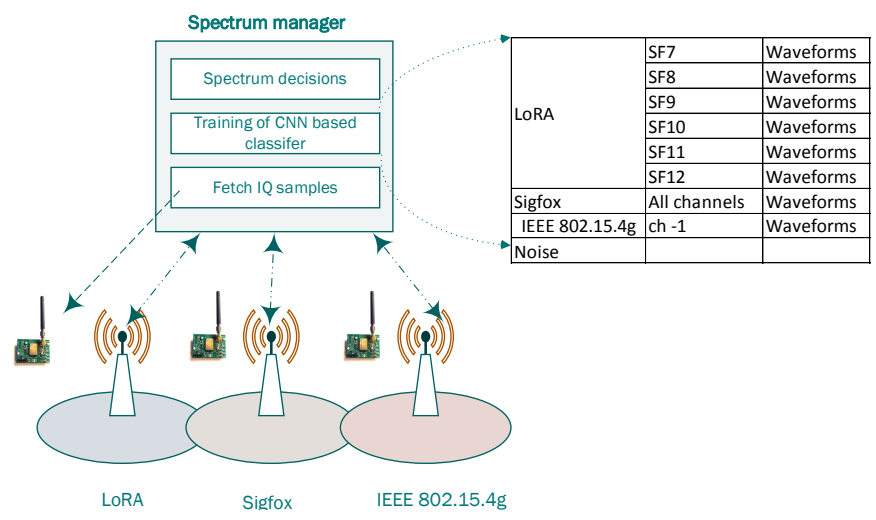

Fig. 1: Proposed spectrum manager framework. 
TABLE I: CNN architecture.

\begin{tabular}{|llll|}
\hline layer & Input size & Parameters & $\begin{array}{l}\text { Activation } \\
\text { function }\end{array}$ \\
\hline $\begin{array}{l}\text { Convolutional } \\
\text { layer }\end{array}$ & $2 \times 500$ & $\begin{array}{l}2 \times 3 \text { filter kernel } \\
64 \text { feature maps } \\
\text { dropout } 60 \%\end{array}$ & Rectified layer \\
$\begin{array}{l}\text { Convolutional } \\
\text { layer }\end{array}$ & $2 \times 496 \times 64$ & $\begin{array}{l}1 \times 3 \text { filter kernel } \\
16 \text { feature maps } \\
\text { dropout } 60 \%\end{array}$ & Rectified layer \\
Dense layer & $1 \times 1015936$ & $\begin{array}{l}500 \text { neurons } \\
\text { Dropout } 60 \%\end{array}$ & Rectified layer \\
Dense layer & $1 \times 500$ & neurons & Softmax \\
\hline
\end{tabular}

\section{FRAMEWORK AND DATA COLLECTION}

The framework that we used for the spectrum manager is shown in Fig. 1. The spectrum manager performs three tasks in the listed order: (i) fetch IQ samples, (ii) identify technologies from the trained model, and (iii) spectrum decisions. In this work, we focus only on the identification task. Both the proposed classifiers are trained offline with all possible waveforms of the technologies. Specifically, the CNN based on IQ samples and the CNN based on FFT are trained with raw IQ samples and their corresponding FFT values, respectively of 9 signal classes: 6 classes of LoRA with spreading factors (SFs) $7,8,9,10,11$, and 12 (each of bandwidth $125 \mathrm{Khz}$ ), 1 class of Sigfox with transmission on all channels (each of bandwidth $100 \mathrm{~Hz}), 1$ class of IEEE 802.15.4g with transmission on one channel (each of bandwidth $600 \mathrm{KHz}$ ), and 1 class of noise. The IQ samples were collected using a setup consisting of a mini B200 USRP and three transmitters (one for each technology). For each signal class, we fetched IQ samples at a samping rate of $1 \mathrm{MHz}$ for $120 \mathrm{sec}(120,000,000$ samples/sec).

\section{CNN BASED CLASSIFIER ACCURACY}

In order to make the IQ samples and their corresponding FFT values compatible with the CNN, we divide them into sensing snapshots, each of duration $500 \mu$ or 500 IQ samples/FFT values. The sensing shot was carefully chosen to take into account the minimum symbol length of the technologies and to minimize the complexity of the CNN. We use a similar structure for both the classifiers which is shown in Table 1.

The proposed classifiers were implemented in Keras with Tensorflow as a backend. The training was done on a highend GPU, i.e., GTX1080Ti. The classification accuracy performance of both the classifiers with respect to SNR (in $\mathrm{dB}$ ) is shown in Fig. 2. For low SNR, the CNN based on FFT performs significantly better compared to its counterpart, whereas for higher SNR conditions the difference is much smaller. This is due to the fact that the frequency domain representation is less prone to noise as compared to its time equivalent.

\section{Demo Setup}

The setup that is used for the demo is shown in Fig. 3. The setup is shown on the left side of the figure: four transmitting radios $(802.15 .4 \mathrm{~g}$, Sigfox, LoRA - SF7, and LoRA SF-12), and a USRP. On the right side of the figure,

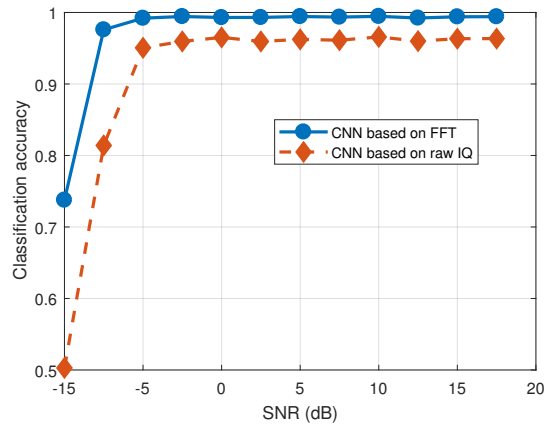

Fig. 2: Classification accuracy.

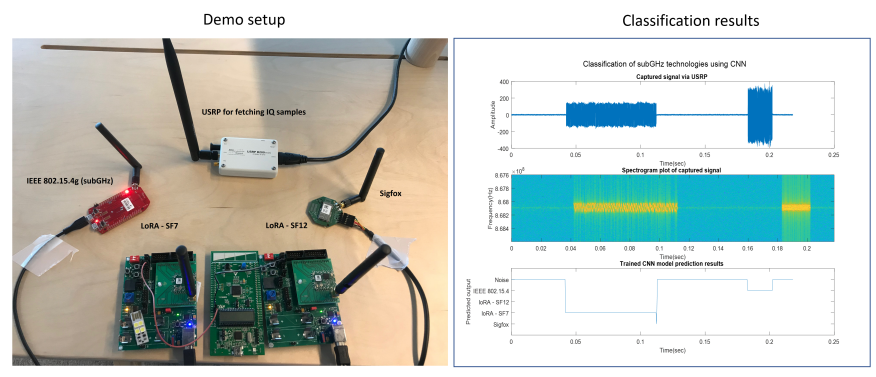

Fig. 3: Demo setup.

the captured IQ samples are validated with the train model and the identification results are shown accordingly in a live format.

\section{CONCLUSION}

This work demonstrates CNN mechanisms toward identification of LPWAN technologies which do not require domain expertise. Most of LPWAN technologies operate in unlicensed bands and their transmission can interfere with each other. In order to minimize the impact of interference, it is important to have a flexible and robust classifier in a spectrum manager, which we demonstrate in this work. To consider low and high SNR environments, we propose two classifiers (i) CNN based on raw IQ samples, and (ii) CNN based on FFT, respectively. We demonstrate the classifiers for 9 different signal classes but they can be extended to any number of classes.

\section{REFERENCES}

[1] https://youtu.be/AvJ_V-fL8z8.

[2] “Cisco," https://www.cisco.com/c/dam/en/us/products/collateral/se/internetof-things/at-a-glance-c45-731471.pdf.

[3] "LoRa," https://www.lora-alliance.org/.

[4] "Sigfox," https://www.sigfox.com.

[5] U. Raza, P. Kulkarni, and M. Sooriyabandara, "Low power wide area networks: An overview," IEEE Communications Surveys \& Tutorials, vol. 19, no. 2, pp. 855-873, 2017.

[6] E. Recommendation, "70-03 relating to the use of short range devices (srd)," Version of, vol. 18, 2009.

[7] K. Kim, I. A. Akbar, K. K. Bae, J.-S. Um, C. M. Spooner, and J. H. Reed, "Cyclostationary approaches to signal detection and classification in cognitive radio," in New frontiers in dynamic spectrum access networks, 2007. DySPAN 2007. 2nd IEEE international symposium on. IEEE, 2007, pp. 212-215.

[8] D. Cireşan, U. Meier, and J. Schmidhuber, "Multi-column deep neural networks for image classification," arXiv preprint arXiv:1202.2745, 2012.

[9] T. J. O'Shea, J. Corgan, and T. C. Clancy, "Convolutional radio modulation recognition networks," in International conference on engineering applications of neural networks. Springer, 2016, pp. 213-226. 